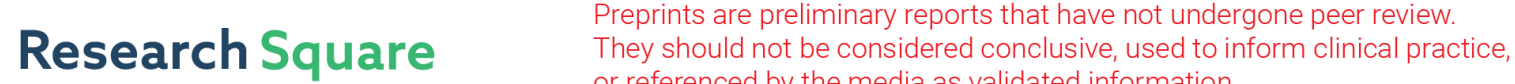 or referenced by the media as validated information. \\ Genes, pathways and networks responding to drought stress in oil palm roots
}

\section{Le Wang}

Temasek Life Sciences Laboratory Ltd

\section{May Lee}

Temasek Life Sciences Laboratory Ltd

baoqing Ye

Temasek Life Sciences Laboratory Ltd

Gen Hua Yue ( $\nabla$ genhua@tll.org.sg )

Temasek Life Sciences Laboratory Ltd https://orcid.org/0000-0002-3537-2248

\section{Research}

Keywords: transcriptome, drought, ion transport, plant hormone, $A B C$ transporters, oil palm

Posted Date: May 23rd, 2020

DOI: https://doi.org/10.21203/rs.3.rs-29882/v1

License: (c) (i) This work is licensed under a Creative Commons Attribution 4.0 International License. Read Full License 


\section{Abstract}

Background: Palm oil is an important feedstock for biofuel. Palm oil yield is seriously affected by drought stress. However, not much is known about the molecular responses of oil palm to drought stress.

Results: We studied the root transcriptomic responses of oil palm seedlings under normal culture and 14day drought stress using RNA-seq and bioinformatics analysis. We identified 1293 differentially expressed genes (DEGs), involved in several molecular processes, such as cell wall biogenesis and functions, phenylpropanoid biosynthesis and metabolisms, ion transport and homeostasis and cellular ketone metabolic process, as well as small molecule biosynthetic process. We observed that DEGs were significantly enriched into the two categories: hormone regulation and metabolism, as well as $A B C$ transporters. In addition, we identified three protein-protein interaction networks involved in the response to drought stress, including ion transport, reactive nitrogen species metabolic process and nitrate assimilation. Finally, 96 differentially expressed transcription factors were detected to be associated with drought stress response, which were classified into 28 families.

Conclusions: The transcriptomic responses of oil palm seedlings to drought stress were systematically analysed, revealing important genes, pathways, networks and transcription factors involved in drought stress responses. These results provide new insights into the mechanisms of drought stress responses in economic crops. The genes and pathways identified in this study provide valuable genomic resources to improve drought tolerance of oil palm by both genetic modification and selective breeding.

\section{Background}

Oil palm (Elaeis guineensis, Jacq.) is the world's most productive oilseed crop, producing approximately $36 \%$ of worldwide vegetable oil [1]. Palm oil is also an important feedstock for producing biofuel [2]. The demand for palm oil is increasing [1]. However, oil palm plantations have been blamed for deforestation and causing the loss of biodiversity [2]. Therefore, continuously increasing plantation area in forests is not an option to increase the production of palm oil. Increasing oil yield in current plantation areas is an optimal way to make the palm oil production sustainable [2]. Oil palm is mainly cultivated in the tropical regions of Southeast Asia, Africa and South America [3, 4]. It needs $\sim 2000 \mathrm{~mm} /$ year rain water to have a normal production and doesn't tolerate drought for more than 90 days [4]. Drought condition affects oil palm in different aspects like photosynthesis rate and growth rate, and reduces its production [5]. Obtaining drought-tolerant oil palm varieties is one of the important targets of genetic improvement of oil palm for sustainable biofuel production [5].

An increasing number of studies have focused on understanding the molecular mechanisms of drought resistance in agronomic plant species or model plant species (e.g. Arabidopsis). These studies have yielded novel knowledge of how plants respond to drought stress with the regulation of critical genes and gene interaction networks [6]. Unfortunately, drought resistance is complex and determined by genetic and environmental factors, and their interactions, making it difficult to increase drought tolerance by 
conventional breeding [7]. Particularly, the mechanisms underlying drought tolerance are highly variable among different species and for some non-model species, it is still poorly understood [8]. Thus, identifying the genetic factors and understanding the underlying mechanisms in different species are essential to improve drought tolerance by gene modifications [9] and breeding [5].

Plants have diverse mechanisms of drought tolerance, to maintain water and ion homeostasis. At the molecular levels, a number of drought-responsive genes and transcription factors have been identified [10]. Some prominent gene classes have been reported to play crucial roles in defending against drought stress [11]. For example, some hormone-related genes can initiate drought resistance signalling [12]; transporters, like $A B C$ transport system, are essential for osmotic signal and osmosensing mechanism [13]; as an important phytohormone, endogenous abscisic acid (ABA) has well-known functions in drought tolerance in plants, by inducing the expression of stress-related genes [14]. Another study has also indicated that jasmonic acid $(\mathrm{JA})$ is associated with stomatal closure to increase drought tolerance, although the mechanisms remain to be elucidated [15]. Other than these genes, transcription factors, like NAC, GmNAC, HD-STARTtype and NF-YB family members, are identified to have played important roles in stress tolerance by regulation of hormone metabolism and hormone signalling pathways [16]. Although many genetic elements and mechanisms have been reported in model plant species and agronomic plant species, in the oil palm, responses to drought stress and the potential mechanism underlying drought tolerance are still poorly understood.

The purpose of the current study was to identify genes, pathways, networks and transcription factors involved in drought response using RNA-seq and bioinformatics analysis to understand more about the mechanism underlying drought tolerance. RNA-seq is a next-generation sequencing technology used to analyse the presence and quantity of RNA molecules in a biological sample [17]. Herein, we challenged oil palm with drought, to simulate frequent drought weather. We further studied the transcriptome responses of roots to drought in the primary tissue for stress signal perception and initiating of cascade gene regulation pathways in response to drought. This study provides both novel insights of molecular mechanisms of tolerance to drought and genomic resources to improve and to develop drought-tolerant oil palms for sustainable production of biofuel.

\section{Results And Discussion}

\section{Morphological and physiological responses to drought}

The overall morphological changes of oil palm seedlings to drought stress with a period of 14 days were observed in both leaves and roots. The effects of drought stress were first observed in leaf morphology, showing initial edge and tip necrosis and then wilting and yellowing for the drought treated samples (Figure 1a). In terms of roots, in comparison to the control, the drought treatments showed significant decrease in the number of roots, root volume and overall biomass (Figure 1b). Trypan blue staining further showed that the drought treatment roots experienced not only obvious cell deformation but also more cell membrane injury than that of the well-watered controls (Figure 1c). Overall, these observations 
are consistent with those of previous studies in plant species under drought stresses and undergoing water deprivation [18]. These results indicate substantial physiological responses of the oil palm seedlings under drought stress [19], and provided useful starting materials to study the genes, pathways and networks involved in drought responses of the important crop species using RNA-seq [20] and bioinformatics analysis.

\section{DEGs in drought responses of oil palm}

An average of 70.2 million (M) cleaned reads were obtained across all six samples. The controls had higher reads coverage than the drought treatments (84.8 M vs 55.7 M) (Supplementary Table S1). Nevertheless, the sequence coverage of the treatments (> 200x of transcriptomes) was sufficient to construct transcripts and identify differentially expressed genes. Approximately $70 \%$ of cleaned reads were uniquely mapped to the reference genome of 31,640 annotated protein coding genes [21]. The drought stressed seedlings showed slightly higher uniquely mapping rates than the controls $(72.1 \% \mathrm{vs}$ $66.3 \%$ ), indicating the duplicated genes also play important roles in response to drought stress [22] in oil palm, a species of palaeotetraploid origin [21] and future studies should also focus on paralogous genes and their potential functions in stress responses [23].

A total of 2,084 and 1,358 DEGs were identified using two different approaches: DESeq2 and EdgeR, respectively, within which 1,293 were shared by the two data sets (Figure 2). In detail, DESeq2 identified 944 down-regulated and 1,140 up-regulated DEGs, while EdgeR screened 624 down-regulated and 734 upregulated DEGs. The number of common down- and up-regulated DEGs were 614 and 679, respectively, between the two approaches (Figure 2 \& Supplementary Table S2). To obtain confident results, only the common DEGS between these two approaches were kept for further analysis. Based on the relative expression of DEGs across samples, the drought treatment and control samples were clearly differentiated by both PCA and hierarchical clustering analyses and showed substantial differences in expression profiles (Figure 3). We further assessed the accuracy of the RNAseq data set by comparing to qPCR of randomly selected nine genes (Supplementary Table S3). We observed an overall high consistency of the expression patterns of these genes between RNA-seq and qPCR (Supplementary Figure 1a), with a correlation coefficient of $0.978(P<0.0001)$, as examined using Pearson's correlation test (Supplementary Figure 1b). In total, these data indicate that the RNA-seq data is solid.

Interestingly, we observed most of the DEGs in subcategories phenylalanine metabolism and tryptophan metabolism to be down-regulated (Table 1). As studied in plant species, phenylalanine and tryptophan metabolisms are more involved in pathogen related immune responses [38, 39]. The down regulation of most DEGs within these categories likely implies the effects of metabolic compensation to drought stress responses by sacrificing the less important biological functions. Except for these enriched pathways, we still found two genes: two-component response regulator ORR9 and two-component response regulator ORR24, that were down- and up-regulated, respectively, were enriched into the subcategory: zeatin biosynthesis (Table 1), which is also revealed to play important roles in drought stress response in 
Populus simonii [40]. Further studies are required to know how the expression patterns of the two genes are related to responses to drought stress in oil palm.

\section{Ontology enrichment analysis of genes responding to drought stress}

To understand the transcriptomic responses to the drought stress, we first carried out gene ontology enrichment analysis using the 1,293 DEGs consistently identified by both EdgeR and DESeq2. A total of $89 \mathrm{GO}$ terms were significantly enriched, involving a number of categories of diverse functions (Supplementary Table S4). The most significant enrichment entities included GO terms related to cell wall biogenesis and functions (e.g., GO: 0009834, G0: 0044036, GO: G0:0009664, GO: 0016998 and GO: 2000652), which was consistent with the above observation that the cell wall of the drought treatments has likely been damaged by severe drought stress and thus has triggered the mechanism of damage and repair (Figure 4). Moreover, we also observed significant enrichments related to phenylpropanoid biosynthesis and metabolisms (e.g., ath00940 and GO: 0009698 and GO: 0046271). It has been widely studied that phenylpropanoid pathway is activated by stress conditions, such as drought, salinity and extreme temperature, and leads to accumulation of phenolic compounds, which play critical physiological roles in regulation under abiotic stress to cope with environmental challenges [24]. Moreover, we found some $\mathrm{GO}$ terms classified into the groups related to ion transport and homeostasis (e.g., GO: 0006811, G0: 0030004 GO: 0030007, G0: 0015698 and GO: 0034220) and response to osmotic stress and water homeostasis (e.g., G0:0006970 and G0: 0030104). Differential expression of genes in these functions likely results from the responses of plants to water deprivation by direct regulation of osmotic pressure $[25,26]$. In addition, a number of genes were enriched into the biological categories related to regulation of cellular ketone metabolic process (GO: 0010565), suggesting that genes involved in ketone metabolic process played important roles in drought stress in oil palm [27, 28]. Hormone regulations are also indispensable to stress responses of plant species. Here, we identified two enriched GO terms related to hormone regulation and metabolism (e.g., GO:0010817 and GO: 0042447). Previous studies have shown that production of numerous secondary metabolites is essential for physiological processes to respond to abiotic stress $[29,30]$. Consistent with these results, we found several significant enrichments related to these terms: small molecule biosynthetic process (GO: 0044283), amino sugar and nucleotide sugar metabolism (ath00520), galactose metabolism (ath00052), benzene-containing compound metabolic process (GO: 0042537), linoleic acid metabolism (ath00591) and xyloglucan metabolic process (GO: 0010411). Interestingly, we also identified significant enriched GO terms, like response to jasmonic acid (G0:0009753) and ABC transporters (ath02010), playing crucial roles in abiotic stress responses (Figure 4).

The interactions of these enriched GO terms were further investigated using network analysis and eight enriched GO networks were identified, with each consisting of no less than 3 genes (Figure 5). The major GO networks involved those related to cell wall related biogenesis and metabolism (GO: 0009834 and GO: 0044036), small molecule related biosynthetic and metabolic processes (ath00940, GO: 0009698, GO: 0044283 and GO: 0010565) and ion transport and homeostasis related processes (GO: 0006811, GO: 0034220 and GO: 0030004). These data imply that genes in these networks are more extensively induced 
to differentially express to respond to drought stress $[25,30,31]$. We further investigated the enriched KEGG pathways and found that the functions of the enriched pathways were generally consistent with that of the enriched GO terms as shown above (Supplementary Table S5). Above all, these enrichment analyses suggest that many genes, pathways and networks respond to the drought stress in the roots of oil palm seedlings. The DEGs, pathways and networks identified in this study provide valuable resources for future studies on their functions to improve drought tolerance of oil palm.

\section{Plant hormone signal transduction in drought stress responses}

Plant hormones not only play crucial roles in controlling growth and development, but also are indispensable in regulation of stress responses [32]. Herein, we first focused on the DEGs involved in plant hormone signal transduction pathway and found significant enrichments of DEGs within subcategories of KEGG pathways including a-Linolenic acid metabolism, carotenoid biosynthesis, phenylalanine metabolism, tryptophan metabolism and zeatin biosynthesis (Table 1). Genes related to aLinolenic acid metabolism have often been revealed to play important roles in drought stress responses $[33,34]$. We found four DEGS were involved in a-Linolenic acid metabolism and three out of them were up-regulated. Interestingly, the down-regulated DEG, jasmonic acid-amido synthetase JAR1 (LOC105048226), was a duplicated copy of the up-regulated one, jasmonic acid-amido synthetase JAR1 (LOC105046997), suggesting functional divergence of paralogous genes since genome duplication events. Nevertheless, consistent with the expression patterns of most DEGs in this subcategory, the upregulated jasmonic acid-amido synthetase JAR1 might be more important in regulation of drought stress response in oil palm. Moreover, we found all of the DEGs, including probable protein phosphatase $2 \mathrm{C} 24$ and two duplicated copies of probable protein phosphatase $2 \mathrm{C} 75$, in the subcategory carotenoid biosynthesis, were up-regulated. These three DEGs were also enriched into the subcategory abscisic acid pathway (ABA) within MAPK signalling pathway (Table 1). Carotenoid biosynthesis signalling pathway is specifically induced by root and contributes to induce $A B A$ production to regulate ion homeostasis, as studied in Arabidoposis [35]. ABA-independent signalling pathways have been widely studied to be involved in the regulation of drought stress response in many kinds of plant species [36]. These results suggest that ABA related genes also play important roles in drought stress responses of oil palm.

\section{$\mathrm{ABC}$ transporters in drought responses}

Membrane transporters play vital roles in regulation of water and ion homeostasis of organisms, among which ATP-binding cassette (ABC) transporters constitute one of the largest protein families and act as both exporters and importers, driven by ATP hydrolysis [37]. An increasing number of studies have shown that $A B C$ transporters play irreplaceable roles in transmembrane allocations of various molecules to adapt to rapidly changing environments, such as water scarcity, heavy metal stress and pathogen stress [38]. In order to survive in these changing abiotic conditions, it is necessary for cells to absorb nutritious chemical substances and discharge endogenous toxins, as well as exchange signalling molecules [38]. Thus, the $A B C$ transporters occupy a diverse range of functions and hence the regulations upon stress responses are also complicated. Here, we found six DEGs were enriched into the pathway of $A B C$ 
transporters (Table 1). Five of them were ABCB subfamily members, among which three (ABC transporter $B$ family member $11, A B C$ transporter $B$ family member 19 and putative multidrug resistance protein (LOC105038824)) were down-regulated and two (ABC transporter B family member 9 and another putative multidrug resistance protein (LOC105060251)) were up-regulated. Such differential expression patterns of these $A B C B$ subfamily transporters indicate the complicated functions in controlling of influx and efflux of chemical molecules $[39,40]$. Other than these $A B C$ transporters, we also identified an $A B C C$ subfamily member, $A B C$ transporter $C$ family member 5 , which was up-regulated. Interestingly, two putative multidrug resistance protein genes (LOC105038824 and LOC105060251) were differentially expressed against drought stress. As shown in previous studies, multidrug resistance-associated proteins are widely involved in regulation of stress responses, such as salt stress, water deprivation, oxidative stress and fungal stress [41]. Taken together, these different types of $A B C$ transporters likely play important roles in responses to drought stress of oil palm.

\section{Protein-protein interaction networks in response to drought responses}

Other than significantly enriched GO terms and KEGG pathways, we also identified three protein-protein interaction networks, focused on ion transport, reactive nitrogen species metabolic process and nitrate assimilation (Figure 6 \& Table 2). Eight DEGs were involved in the ion transport network, among which five (ammonium transporter 2 member 1 (AMT2-1), amino acid transporter ANT1 (ANT1), cation/H(+) antiporter 20 (CHX20), plasma membrane ATPase 4 (PMA4) and potassium channel AKT1 (AKT1)) and three (receptor-like protein kinase HSL1 (HSL1), plasma membrane ATPase (PMA) and ABC transporter G family member 42 (ABCG42)) were up- and down-regulated, respectively (Table 2). Interestingly, most of the cation channel and transporter genes were up-regulated, including ammonium transporter 2 member 1 (AMT1), amino acid transporter ANT1 (ANT1), cation/H(+) antiporter 20 (CHX20) and potassium channel AKT1 (KT1), indicating their positive effects in regulating ion homeostasis in oil palm [42]. Nevertheless, we also observed three DEGs were down-regulated in the same network, implying both positive and negative feedback regulations are acting on this network [43]. Reactive nitrogen species metabolic process is also suggested to have critical roles in stress responses, such as drought and salinity [44]. Consistently, we identified three up-regulated genes: magnesium transporter MRS2-1 (MGT2), putative chloride channel-like protein CLC-g (AT5G33280) and serine/threonine protein kinase OSK1 (KIN10), in this protein-protein interaction network. Nitrate assimilation is another biological process affecting salt and water stress tolerance in plants [45]. Here, we found four DEGs involved in this network: two were up-regulated (cationic amino acid transporter 6, chloroplastic (CAT6) and sodium/hydrogen exchanger 4 (NHX4)), while the other two were down-regulated (amino acid permease 8 (AAP8) and vacuolar cation/proton exchanger 1a (CAX1)). As these protein-protein interaction networks play crucial roles in drought stress response, the DEGs involved in these networks provide excellent candidates to improve drought tolerance of oil palm by genetic engineering and/or selective breeding.

\section{Transcription factors in drought responses}


To date, more and more studies have paid attention to the biological functions of transcription factors as regulatory elements binding proteins [10]. Transcription factors are vital for development, response to intercellular and environmental signals and pathogenesis [10]. The expression changes are often associated with important cellular processes [7]. In this study, we identified 96 differentially expressed transcription factors that were classified into 28 families (Supplementary Table $\mathbf{6} 6$ \& Table 3). Previous studies have shown that transcription factors are broadly involved in drought/abiotic stress responses, such as the members of family MYB, WRKY, DREB, NAC and AP2/EREBP [16, 46-48]. Here we also observed genes in these transcription factor families were differentially expressed against drought stress in oil palm, further supporting their important roles in drought tolerance in plant species. Interestingly, we found several families of transcription factors that were rarely studied and involved in abiotic stress responses, such as the $\mathrm{C} 2 \mathrm{H} 2$, LFY and TALE transcription families. Therefore, it is important to understand the mechanisms of regulatory functions of these genes, which might be useful to help improve drought tolerance of related plant species.

\section{Conclusions}

We investigated transcriptomic response of root against drought stress in oil palm seedlings. We identified over 1000 DEGs responding to the drought stress, including the genes mainly involved in cell wall biogenesis and functions, phenylpropanoid biosynthesis and metabolisms and ion transport and homeostasis. We functionally enriched the genes in plant hormone signal transduction and ABC transporters pathways, which likely have played crucial roles in regulation of water deprivation. Three protein-protein interaction networks were identified that were related to ion transport, reactive nitrogen species metabolic process and nitrate assimilation. We also detected 96 transcription factors that were differentially expressed upon drought stress. The identified DEGs likely play important roles in drought tolerance of oil palm. This study helps understand the mechanism of drought stress response and provides valuable resources for future genetic improvement of drought tolerance in the commercial lines.

\section{Methods}

\section{Plant materials and drought treatment}

Seeds of Tenera palms were geminated with a standard protocol $[4,36]$. The seedlings were grown in a nursery for 120 days before drought stress treatment. Eight oil palm Tenera seedings were planted in pots with diameter of $20 \mathrm{~cm}$ containing natural soil with water content of $23 \%$ ( $2.3 \mathrm{~g}$ water per $10 \mathrm{~g}$ soil), and placed in a greenhouse with a natural tropical temperature ranging from $28^{\circ} \mathrm{C}$ to $34^{\circ} \mathrm{C}, 30-50 \%$ relative humidity and natural photoperiod. Four seedings were watered twice a week to maintain water content of $>23 \%$ while the other four seedlings were used as drought treatment without watering for two weeks. This is to simulate the condition of dry weather occasionally encountered in recent years, with global climate change and warming. After drought stress challenge of 14 days, the root tissues of both the control and experimental groups were harvested and measured, respectively. The samples were then preserved at $-80^{\circ} \mathrm{C}$ for RNA isolation. 


\section{RNA extraction and sequencing}

Total RNA was isolated from roots using RNeasy Plant Mini Kit (Qiagen, Germany), according to the manufacturer's instructions. RNA quality was assessed by agarose gels and concentration was measured by NanoDrop (Thermo Fisher Scientific, USA). One $\mu \mathrm{g}$ total RNA from each sample was firstly treated with RNase-free DNase I (Sigma-Aldrich, Singapore) and then used for mRNA library construction with Illumina TruSeq RNA Library Prep Kit v2 (Illumina, USA), according to the manufacturer's instructions. The libraries were sent to an Illumina NextSeq500 (IIlumina, USA) for paired-end sequencing of $2 \times 75 \mathrm{bp}$. Three biological replicates were sequenced for both control and drought treated samples. For validation of RNA sequencing data using real-time quantitative PCR (qPCR), two $\mu \mathrm{g}$ total RNA was treated with RNase-free DNase I (Sigma-Aldrich, Singapore) and was then used for synthesizing CDNA with the MMLV reverse transcriptase (Promega, USA).

\section{Identification of differentially expressed genes (DEGs)}

Raw sequencing reads were processed using the program process_shortreads in Stacks package [49], to demultiplex samples, filter adaptors and clean up low quality reads. The program STAR [50] was employed to align and map the cleaned reads to the reference genome of oil palm [21], with default parameters. Only uniquely mapped reads were used to analyse the expression patterns of annotated genes. The program HTSeq-count [51] was then used to count the expression level of each annotated gene, based on the information of gene features in the genome annotation file. We used both DESeq2 [52] and EdgeR [53] to normalize the relative expression of transcripts across samples. Only transcripts with the number of counts per million (CPM) mapped reads of $>1$ were retained for further analysis. Transcripts with a fold change (FC) value of $>2$ or $<-2$ and with a significance value of 0.01 after application of Benjamini-Hochberg false discovery rate (FDR) [54] were considered as differentially expressed genes, between drought treatment and control groups. Only DEGs that were consistently identified by both DESeq2 and EdgeR, were used for further analysis.

\section{Functional annotation of DEGs}

Gene Ontology (GO) and Kyoto Encyclopedia of Genes and Genomes (KEGG) accessions were retrieved for each DEG, according to the PalmXplore database of oil palm [55]. We firstly clustered all the samples using both principal component analysis (PCA) and heatmap approaches with the program ClustVis [56], based on the relative expression of DEGs, to investigate the overall expression patterns between drought treatment and control groups. Gene ontology enrichment analysis was carried out using the program Metascape [57]. The Metascape program [57] was further employed to study the protein-protein interactions using network analysis by referencing to Arabidopsis. The candidate signalling pathways associated DEGs were classified and enriched by annotating against the Kyoto Encyclopaedia of Genes and Genomes (KEGG) database [58] of oil palm.

\section{Validation of RNA-seq data using qPCR}


DEGs were randomly selected and the relative expression patterns revealed by RNA-seq were examined by qPCR, to assess the effectiveness and accuracy of the whole DEGs dataset. Primers of randomly selected DEGs were designed according to the coding sequences, obtained from the annotated reference genome, using the program Primer3 [59]. Both $\beta$-actin gene and glyceraldehyde 3-phosphate dehydrogenase gene (GAPDH) were used as housekeeping genes to normalize the relative expression of genes, according to our previous study [60]. The $2-\Delta \triangle C T$ method was used to quantify the expression level according to our previous method [61]. The experiment was carried out with three biological replications, each with three technical replicates.

\section{Abbreviation}

CPO: Crude palm oil; DEG: differentially expressed gene; PCR: polymerase chain reaction; qRT-PCR: quantitative reverse transcription PCR; ABC transporters: The ATP-binding cassette transporters; RNA-seq: RNA sequencing; GO: Gene Ontology; KEGG: Kyoto Encyclopedia of Genes and Genomes; ABA: Abscisic acid pathway; MAPK: Mitogen-activated protein kinase; ABCB: $B$ family of the ATP-binding cassette; MYB: MYB Transcription factors; CPM: Counts per million, FC: Fold of change; qPCR: Quantitative PCR.

\section{Declaration}

\section{Ethics approval and consent to participate}

Not applicable.

\section{Consent for publication}

Not applicable.

\section{Competing interests}

The authors declare that they have no competing interests.

\section{Funding}

This research was supported by the Internal Funds of the Temasek Life Sciences Lab (9200).

\section{Authors' contributions}

GHY designed the study. ML and BY challenged samples and constructed libraries. ML sequenced the libraries. LW carried out qPCR. LW analysed the data. LW and GHY drafted the manuscript. All authors read and approved the final manuscript.

\section{Acknowledgements}

We thank Dr Zi Yi Wan and the intern ZY Sam for technical assisting in the experiments. 
Availability of data and material

Raw sequencing reads used in this study have been deposited to the NCBI SRA database with an accession no. PRJDB9517.

\section{References}

1. Palm oil production [https://www.palmoilandfood.eu/en/palm-oil-production]

2. Gatti RC, Liang J, Velichevskaya A, Zhou M: Sustainable palm oil may not be so sustainable. Sci Total Environ. 2019, 652:48-51.

3. Zhang C, Peng X, Guo XF, Tang GJ, Sun FL, Liu SD, Xi YJ: Transcriptional and physiological data reveal the dehydration memory behavior in switchgrass (Panicum virgatum L.). Biotechnol Biofuels. 2018, 11.

4. Corley RHV, Tinker PB: The oil palm. Oxford: John Wiley \& Sons; 2008.

5. Corley R, Rao V, Palat T, Praiwan T: Breeding for drought tolerance in oil palm. J Oil Palm Res. 2017, 30:26-35.

6. Zhang JZ, Creelman RA, Zhu J-K: From laboratory to field. Using information from Arabidopsis to engineer salt, cold, and drought tolerance in crops. Plant Physiol. 2004, 135(2):615-621.

7. Golldack D, Lüking I, Yang O: Plant tolerance to drought and salinity: stress regulating transcription factors and their functional significance in the cellular transcriptional network. Plant Cell Rep. 2011, 30(8):1383-1391.

8. Sinclair TR: Challenges in breeding for yield increase for drought. Trends Plant Sci. 2011, 16(6):289293.

9. Mittler R, Blumwald E: Genetic engineering for modern agriculture: challenges and perspectives. Annu Rev Plant Biol. 2010, 61:443-462.

10. Joshi R, Wani SH, Singh B, Bohra A, Dar ZA, Lone AA, Pareek A, Singla-Pareek SL: Transcription factors and plants response to drought stress: current understanding and future directions. Frontiers Plant Sci. 2016, 7:1029.

11. Umezawa T, Fujita M, Fujita Y, Yamaguchi-Shinozaki K, Shinozaki K: Engineering drought tolerance in plants: discovering and tailoring genes to unlock the future. Curr Opin Biotechnol. 2006, 17(2):113122.

12. Ko JH, Yang SH, Han KH: Upregulation of an Arabidopsis RING-H2 gene, XERICO, confers drought tolerance through increased abscisic acid biosynthesis. Plant J. 2006, 47(3):343-355.

13. Kuromori T, Sugimoto E, Shinozaki K: Arabidopsis mutants of AtABCG22, an ABC transporter gene, increase water transpiration and drought susceptibility. Plant J. 2011, 67(5):885-894.

14. luchi S, Kobayashi M, Taji T, Naramoto M, Seki M, Kato T, Tabata S, Kakubari Y, YamaguchiShinozaki K, Shinozaki K: Regulation of drought tolerance by gene manipulation of 9-cis- 
epoxycarotenoid dioxygenase, a key enzyme in abscisic acid biosynthesis in Arabidopsis. Plant J. 2001, 27(4):325-333.

15. Alam MM, Nahar K, Hasanuzzaman M, Fujita M: Exogenous jasmonic acid modulates the physiology, antioxidant defense and glyoxalase systems in imparting drought stress tolerance in different Brassica species. Plant Biotchnol Rep. 2014, 8(3):279-293.

16. Huang Q, Wang Y, Li B, Chang J, Chen M, Li K, Yang G, He G: TaNAC29, a NAC transcription factor from wheat, enhances salt and drought tolerance in transgenic Arabidopsis. BMC Plant Biol. 2015, 15(1):268.

17. Abdullah HM, Chhikara S, Akbari P, Schnell DJ, Pareek A, Dhankher OP: Comparative transcriptome and metabolome analysis suggests bottlenecks that limit seed and oil yields in transgenic Camelina sativa expressing diacylglycerol acyltransferase 1 and glycerol-3-phosphate dehydrogenase. Biotechnol Biofuels. 2018, 11.

18. Varshney RK, Tuberosa R, Tardieu F: Progress in understanding drought tolerance: from alleles to cropping systems. In.: Oxford University Press UK; 2018.

19. Shao H-B, Chu L-Y, Jaleel CA, Zhao C-X: Water-deficit stress-induced anatomical changes in higher plants. CR Biol. 2008, 331(3):215-225.

20. Ozsolak F, Milos PM: RNA sequencing: advances, challenges and opportunities. Nat Rev Genet. 2011, 12(2):87-98.

21. Singh R, Ong-Abdullah M, Low E-TL, Manaf MAA, Rosli R, Nookiah R, Ooi LC-L, Ooi SE, Chan K-L, Halim MA: Oil palm genome sequence reveals divergence of interfertile species in Old and New worlds. Nature. 2013, 500(7462):335-339.

22. Zou C, Lehti-Shiu MD, Thomashow M, Shiu S-H: Evolution of stress-regulated gene expression in duplicate genes of Arabidopsis thaliana. PLoS Genet. 2009, 5(7):e1000581.

23. Teotia S, Lamb RS: The paralogous genes RADICAL-INDUCED CELL DEATH1 and SIMILAR TO RCD ONE1 have partially redundant functions during Arabidopsis development. Plant Physiol. 2009, 151(1):180-198.

24. Dixon RA, Paiva NL: Stress-induced phenylpropanoid metabolism. Plant Cell. 1995, 7(7):1085-1097.

25. Zhu J-K: Regulation of ion homeostasis under salt stress. Curr Opin Plant Biol. 2003, 6(5):441-445.

26. Zhu JK: Salt and drought stress signal transduction in plants. Annu Rev Plant Biol. 2002, 53(1):247273.

27. Ranjan A, Pandey N, Lakhwani D, Dubey NK, Pathre UV, Sawant SV: Comparative transcriptomic analysis of roots of contrasting Gossypium herbaceum genotypes revealing adaptation to drought. BMC Genomics. 2012, 13(1):680.

28. Zeng X, Bai L, Wei Z, Yuan H, Wang Y, Xu Q, Tang Y, Nyima T: Transcriptome analysis revealed the drought-responsive genes in Tibetan hulless barley. BMC Genomics. 2016, 17(1):386.

29. Niinemets Ü: Uncovering the hidden facets of drought stress: secondary metabolites make the difference. Tree Physiol. 2016, 36(2):129-132. 
30. Akula R, Ravishankar GA: Influence of abiotic stress signals on secondary metabolites in plants. Plant Signaling \& Behavior. 2011, 6(11):1720-1731.

31. Safronov O, Kreuzwieser J, Haberer G, Alyousif MS, Schulze W, Al-Harbi N, Arab L, Ache P, Stempfl T, Kruse J: Detecting early signs of heat and drought stress in Phoenix dactylifera (date palm). PLoS ONE. 2017, 12(6):e0177883.

32. Daszkowska-Golec A, Szarejko I: Open or close the gate-stomata action under the control of phytohormones in drought stress conditions. Frontiers Plant Sci. 2013, 4:138.

33. Upchurch RG: Fatty acid unsaturation, mobilization, and regulation in the response of plants to stress. Biotechnology Letters. 2008, 30(6):967-977.

34. León J, Sánchez-Serrano JJ: Molecular biology of jasmonic acid biosynthesis in plants. Plant Physiology and Biochemistry. 1999, 37(5):373-380.

35. Ruiz-Sola MÁ, Arbona V, Gómez-Cadenas A, Rodríguez-Concepción M, Rodríguez-Villalón A: A root specific induction of carotenoid biosynthesis contributes to ABA production upon salt stress in Arabidopsis. PLoS ONE. 2014, 9(3):e90765.

36. Yoshida T, Mogami J, Yamaguchi-Shinozaki K: ABA-dependent and ABA-independent signaling in response to osmotic stress in plants. Curr Opin Plant Biol. 2014, 21:133-139.

37. Broehan $\mathrm{G}$, Kroeger T, Lorenzen M, Merzendorfer $\mathrm{H}$ : Functional analysis of the ATP-binding cassette (ABC) transporter gene family of Tribolium castaneum. BMC Genomics. 2013, 14(1):6.

38. Martinoia E, Klein M, Geisler M, Bovet L, Forestier C, Kolukisaoglu U, MuÈller-RoÈber B, Schulz B: Multifunctionality of plant ABC transporters-more than just detoxifiers. Planta. 2002, 214(3):345355.

39. Yazaki K: ABC transporters involved in the transport of plant secondary metabolites. FEBS Lett. 2006, 580(4):1183-1191.

40. Cho $\mathrm{M}$, Cho H: The function of ABCB transporters in auxin transport. Plant Signaling \& Behavior. 2013, 8(2):642-654.

41. Wolfger $\mathrm{H}$, Mamnun $\mathrm{YM}$, Kuchler $\mathrm{K}$ : Fungal $A B C$ proteins: pleiotropic drug resistance, stress response and cellular detoxification. Research in Microbiology. 2001, 152(3-4):375-389.

42. Osakabe Y, Arinaga N, Umezawa T, Katsura S, Nagamachi K, Tanaka H, Ohiraki H, Yamada K, Seo S$\mathrm{U}, \mathrm{Abo} \mathrm{M}$ : Osmotic stress responses and plant growth controlled by potassium transporters in Arabidopsis. Plant Cell. 2013, 25(2):609-624.

43. Valliyodan B, Nguyen HT: Understanding regulatory networks and engineering for enhanced drought tolerance in plants. Curr Opin Plant Biol. 2006, 9(2):189-195.

44. Filippou P, Bouchagier P, Skotti E, Fotopoulos V: Proline and reactive oxygen/nitrogen species metabolism is involved in the tolerant response of the invasive plant species Ailanthus altissima to drought and salinity. Environmental and Experimental Botany. 2014, 97:1-10.

45. Azcón R, Tobar RM: Activity of nitrate reductase and glutamine synthetase in shoot and root of mycorrhizal Allium cepa: effect of drought stress. Plant Sci. 1998, 133(1):1-8. 
46. Baldoni E, Genga A, Cominelli E: Plant MYB transcription factors: their role in drought response mechanisms. Intern J Mol Sci. 2015, 16(7):15811-15851.

47. Tripathi P, Rabara RC, Rushton PJ: A systems biology perspective on the role of WRKY transcription factors in drought responses in plants. Planta. 2014, 239(2):255-266.

48. Liu Q, Zhao N, Yamaguch-Shinozaki K, Shinozaki K: Regulatory role of DREB transcription factors in plant drought, salt and cold tolerance. Chin Sci Bull. 2000, 45(11):970-975.

49. Catchen J, Hohenlohe PA, Bassham S, Amores A, Cresko WA: Stacks: an analysis tool set for population genomics. Mol Ecol. 2013, 22(11):3124-3140.

50. Dobin A, Davis CA, Schlesinger F, Drenkow J, Zaleski C, Jha S, Batut P, Chaisson M, Gingeras TR: STAR: ultrafast universal RNA-seq aligner. Bioinformatics. 2013, 29(1):15-21.

51. Anders S, Pyl PT, Huber W: HTSeq-a Python framework to work with high-throughput sequencing data. Bioinformatics. 2015, 31(2):166-169.

52. Love MI, Huber W, Anders S: Moderated estimation of fold change and dispersion for RNA-seq data with DESeq2. Genome Biol. 2014, 15(12):550.

53. Robinson MD, McCarthy DJ, Smyth GK: edgeR: a Bioconductor package for differential expression analysis of digital gene expression data. Bioinformatics. 2010, 26(1):139-140.

54. Thissen D, Steinberg L, Kuang D: Quick and easy implementation of the Benjamini-Hochberg procedure for controlling the false positive rate in multiple comparisons. J Edu Behav Statis. 2002, 27(1):77-83.

55. Sanusi NSNM, Rosli R, Halim MAA, Chan K-L, Nagappan J, Azizi N, Amiruddin N, Tatarinova TV, Low E-TL: PalmXplore: oil palm gene database. Database. 2018, 2018:bay095.

56. Metsalu T, Vilo J: ClustVis: a web tool for visualizing clustering of multivariate data using Principal Component Analysis and heatmap. Nucleic Acids Res. 2015, 43(W1):W566-W570.

57. Zhou Y, Zhou B, Pache L, Chang M, Khodabakhshi AH, Tanaseichuk O, Benner C, Chanda SK: Metascape provides a biologist-oriented resource for the analysis of systems-level datasets. Nature Commun. 2019, 10(1):1-10.

58. Kanehisa M, Furumichi M, Tanabe M, Sato Y, Morishima K: KEGG: new perspectives on genomes, pathways, diseases and drugs. Nucleic Acids Res. 2017, 45(D1):D353-D361.

59. Untergasser A, Cutcutache I, Koressaar T, Ye J, Faircloth BC, Remm M, Rozen SG: Primer3-new capabilities and interfaces. Nucleic Acids Res. 2012, 40(15):e115-e115.

60. Zhang Y, Bai B, Lee M, Alfiko Y, Suwanto A, Yue GH: Cloning and characterization of EgGDSL, a gene associated with oil content in oil palm. Sci Rep. 2018, 8(1):1-11.

61. Wang L, Liu P, Wan ZY, Huang SQ, Wen YF, Lin G, Yue GH: RNA-Seq revealed the impairment of immune defence of tilapia against the infection of Streptococcus agalactiae with simulated climate warming. Fish Shellfish Immunol. 2016, 55:679-689.

\section{Tables}


Table 1 Selected enriched KEGG pathways and DEGs in response to drought challenge in the roots of oil palm seedlings.

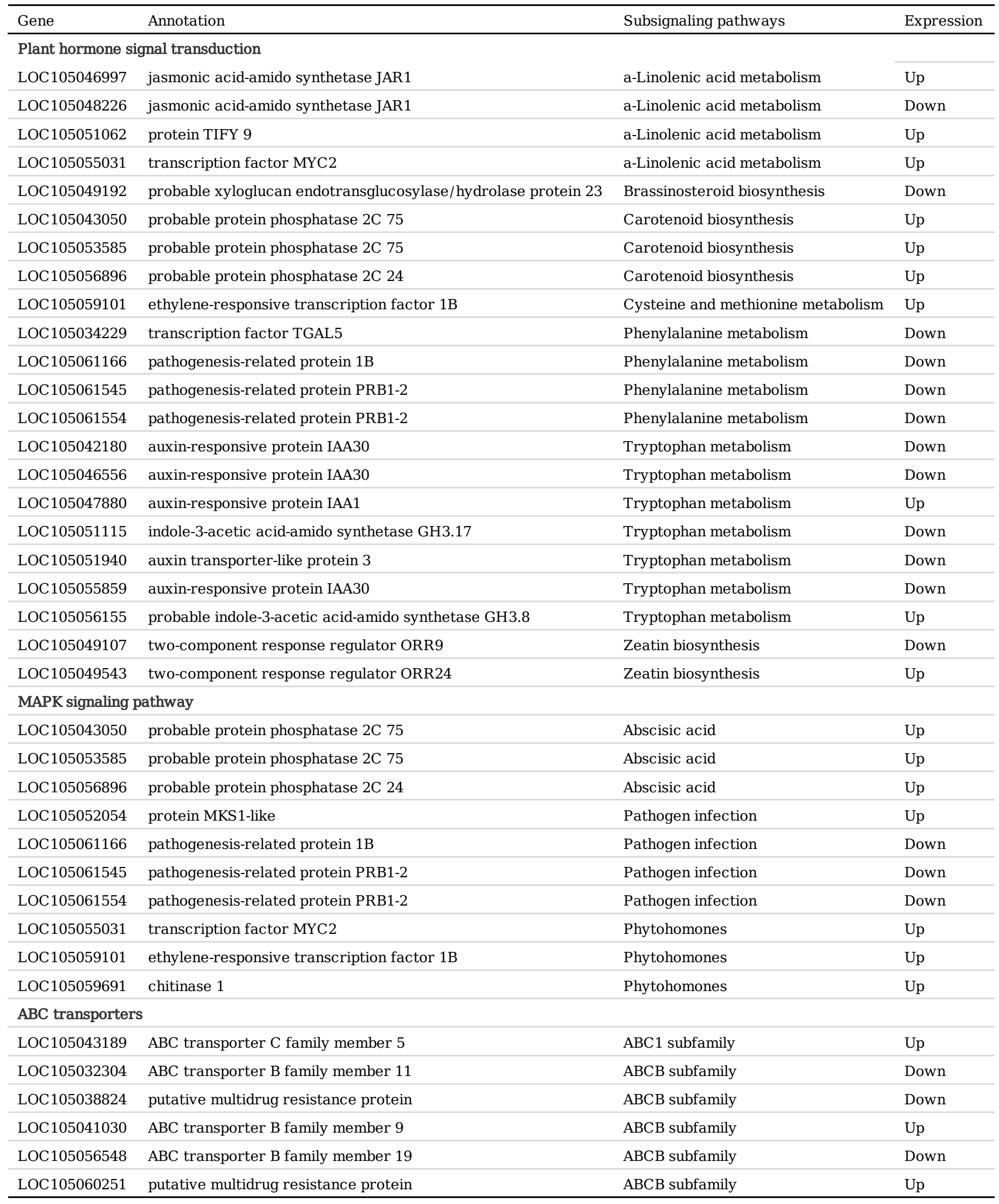


Table 2 DEGs in three enriched protein-protein interaction networks in response to drought challenge in the roots of oil palm seedlings.

\begin{tabular}{|c|c|c|}
\hline Gene & Annotation & Expression \\
\hline \multicolumn{3}{|l|}{ Ion transport } \\
\hline LOC105045862 & ammonium transporter 2 member 1 & Up \\
\hline LOC105049578 & amino acid transporter ANT1 & Up \\
\hline LOC105037902 & receptor-like protein kinase HSL1 & Down \\
\hline LOC105033828 & cation/H(+) antiporter 20 & Up \\
\hline LOC105039277 & plasma membrane ATPase & Down \\
\hline LOC105052943 & plasma membrane ATPase 4 & Up \\
\hline LOC105047848 & potassium channel AKT1 & Up \\
\hline LOC105040536 & ABC transporter $\mathrm{G}$ family member 42 & Down \\
\hline \multicolumn{3}{|c|}{ Reactive nitrogen species metabolic process } \\
\hline LOC105060165 & magnesium transporter MRS2-1 & Up \\
\hline LOC105050121 & putative chloride channel-like protein CLC-g & Up \\
\hline LOC105044259 & serine/threonine protein kinase OSK1 & Up \\
\hline \multicolumn{3}{|c|}{ Nitrate assimilation } \\
\hline LOC105037451 & amino acid permease 8 & Down \\
\hline LOC105052094 & cationic amino acid transporter 6 , chloroplastic & Up \\
\hline LOC105032563 & vacuolar cation/proton exchanger 1a & Down \\
\hline LOC105038946 & sodium/hydrogen exchanger 3 & Up \\
\hline
\end{tabular}

Table 3 Categorization of differentially expressed transcription factors and the patterns of their expressions after drought stress in the roots of oil palm seedlings. 


\begin{tabular}{|c|c|c|c|}
\hline Family & Number & Down & Up \\
\hline AP2 & 2 & 1 & 1 \\
\hline ARR-B & 2 & 0 & 2 \\
\hline B3 & 3 & 0 & 3 \\
\hline bHLH & 10 & 4 & 6 \\
\hline bZIP & 5 & 2 & 3 \\
\hline $\mathrm{C} 2 \mathrm{H} 2$ & 5 & 1 & 4 \\
\hline $\mathrm{C} 3 \mathrm{H}$ & 1 & 0 & 1 \\
\hline Dof & 1 & 0 & 1 \\
\hline E2F/DP & 1 & 0 & 1 \\
\hline ERF & 9 & 1 & 8 \\
\hline FAR1 & 1 & 0 & 1 \\
\hline G2-like & 2 & 1 & 1 \\
\hline GATA & 1 & 0 & 1 \\
\hline GRAS & 2 & 0 & 2 \\
\hline GRF & 1 & 1 & 0 \\
\hline HB-other & 1 & 0 & 1 \\
\hline HD-ZIP & 6 & 3 & 3 \\
\hline HSF & 3 & 0 & 3 \\
\hline LBD & 1 & 0 & 1 \\
\hline LFY & 1 & 0 & 1 \\
\hline MYB & 15 & 9 & 6 \\
\hline NAC & 17 & 9 & 8 \\
\hline RAV & 1 & 0 & 1 \\
\hline TALE & 1 & 0 & 1 \\
\hline TCP & 1 & 0 & 1 \\
\hline Trihelix & 1 & 0 & 1 \\
\hline WRKY & 1 & 0 & 1 \\
\hline YABBY & 1 & 0 & 1 \\
\hline Total & 96 & 32 & 64 \\
\hline
\end{tabular}

Figures 

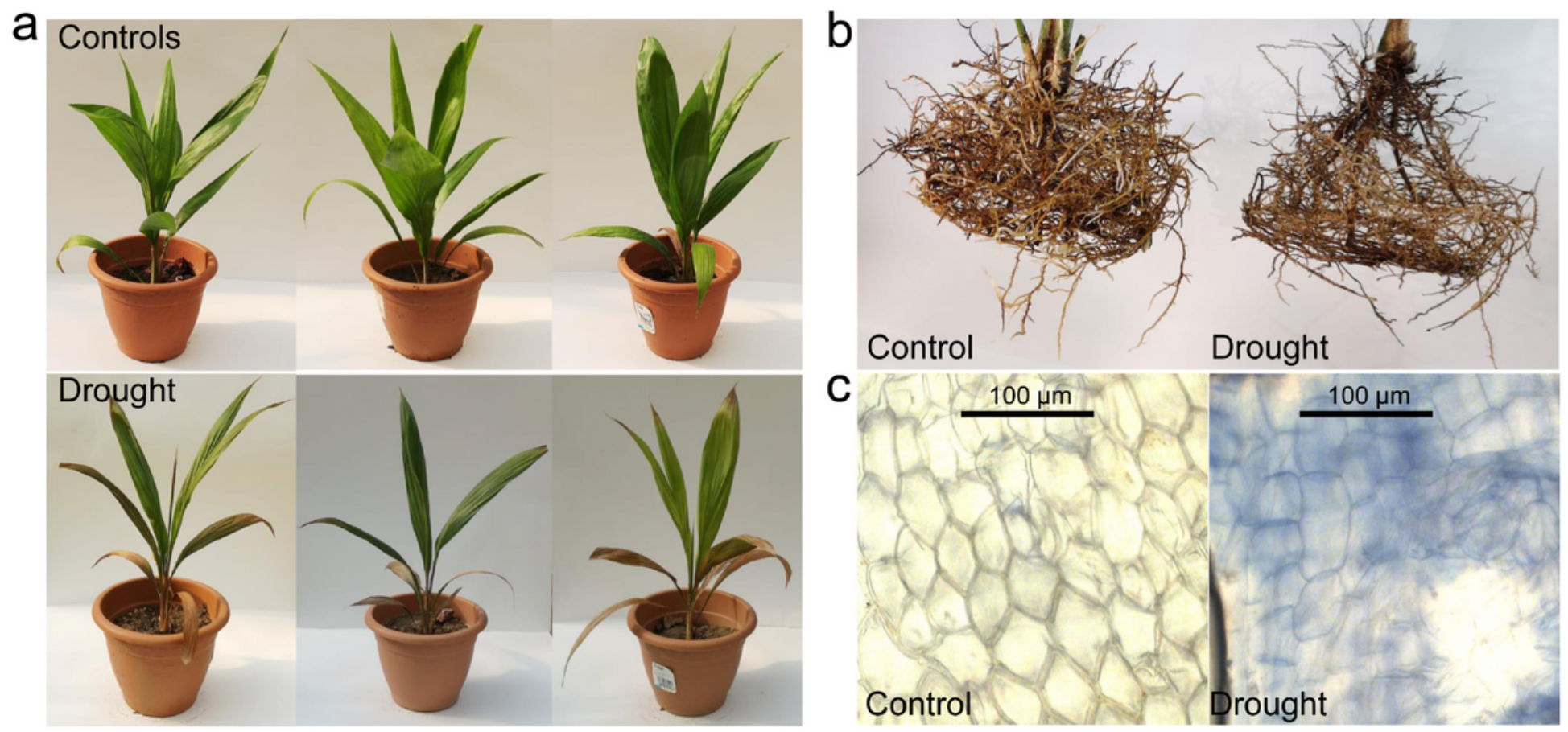

Figure 1

Phenotypic changes of oil palm seedlings to drought stress. The leaves' (a) and roots' (b) responses to severe drought stress in comparison to controls at 10 days post challenge, and (c) trypan blue staining of roots 
a

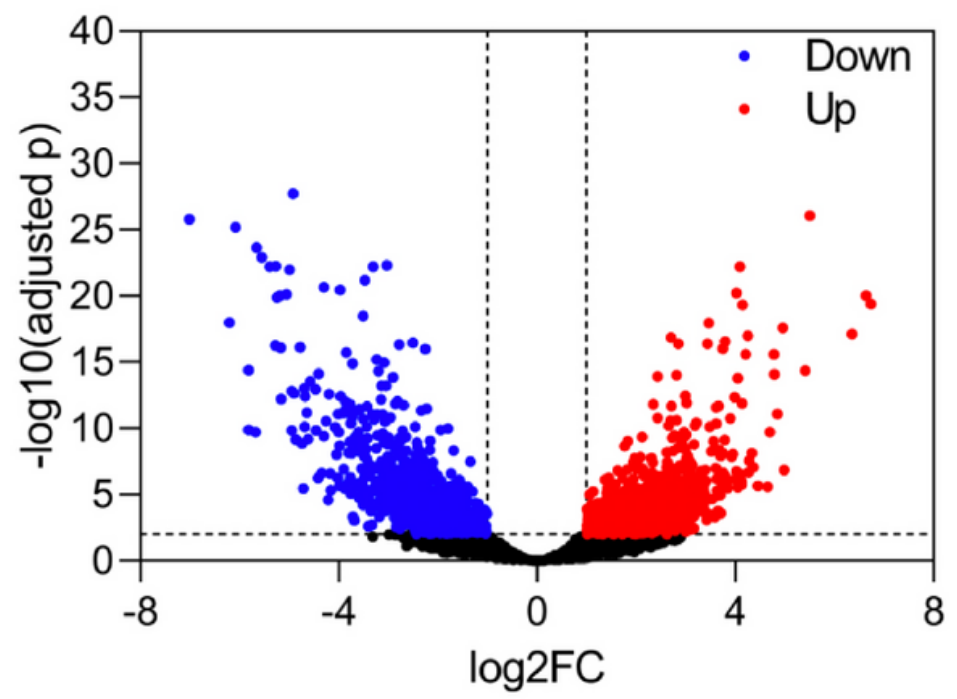

b

EdgeR
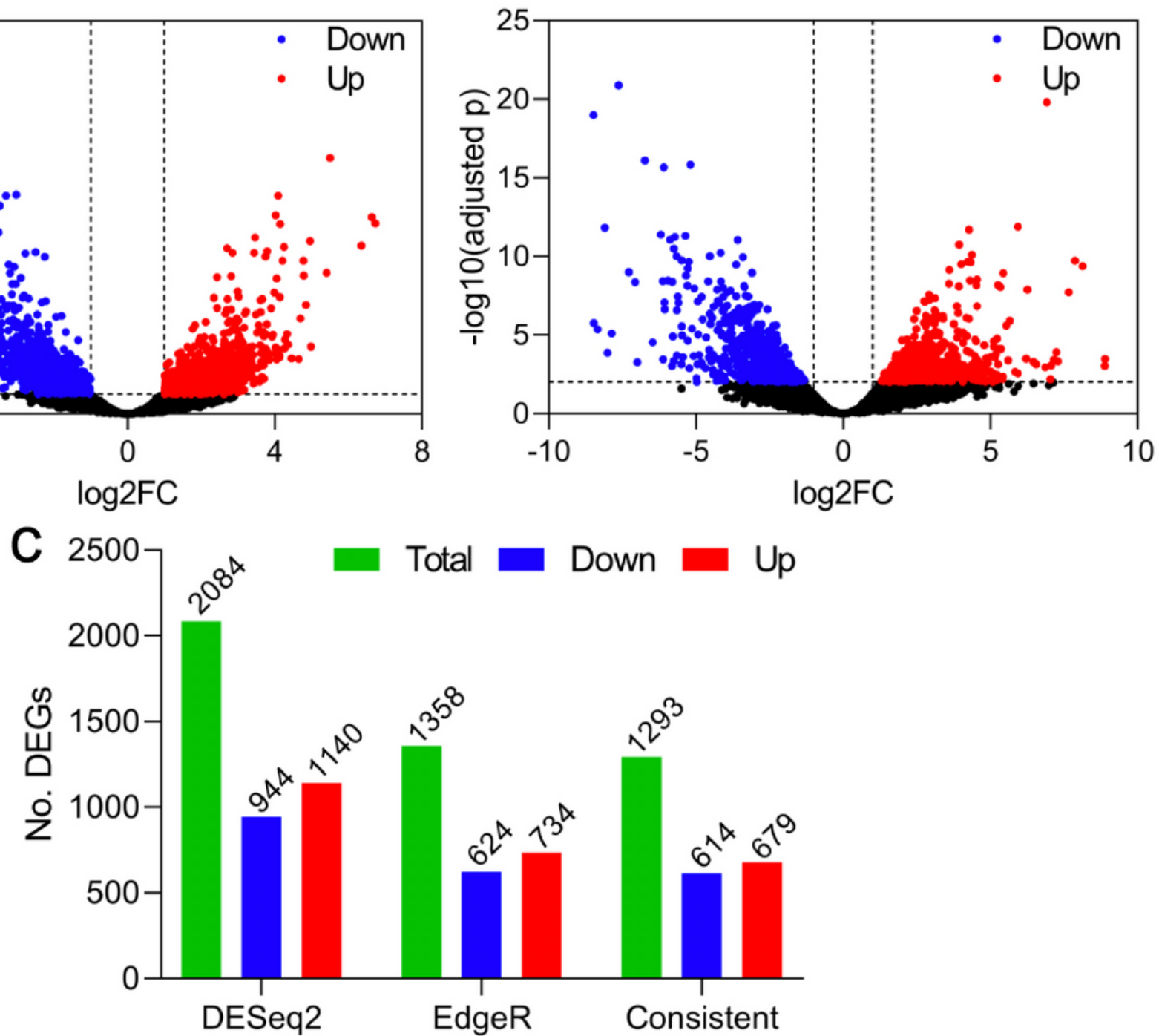

Figure 2

Comparison of differentially expressed genes (DEGs) in the roots of oil palm seedlings under drought stress, as shown by Volcano plot, identified by DESeq2 (a) and EdgeR (b). The numbers of DEGs that are down-regulated and up-regulated revealed by DESeq2 and EdgeR and the consistent DEGs between the two methods are shown in (c). 

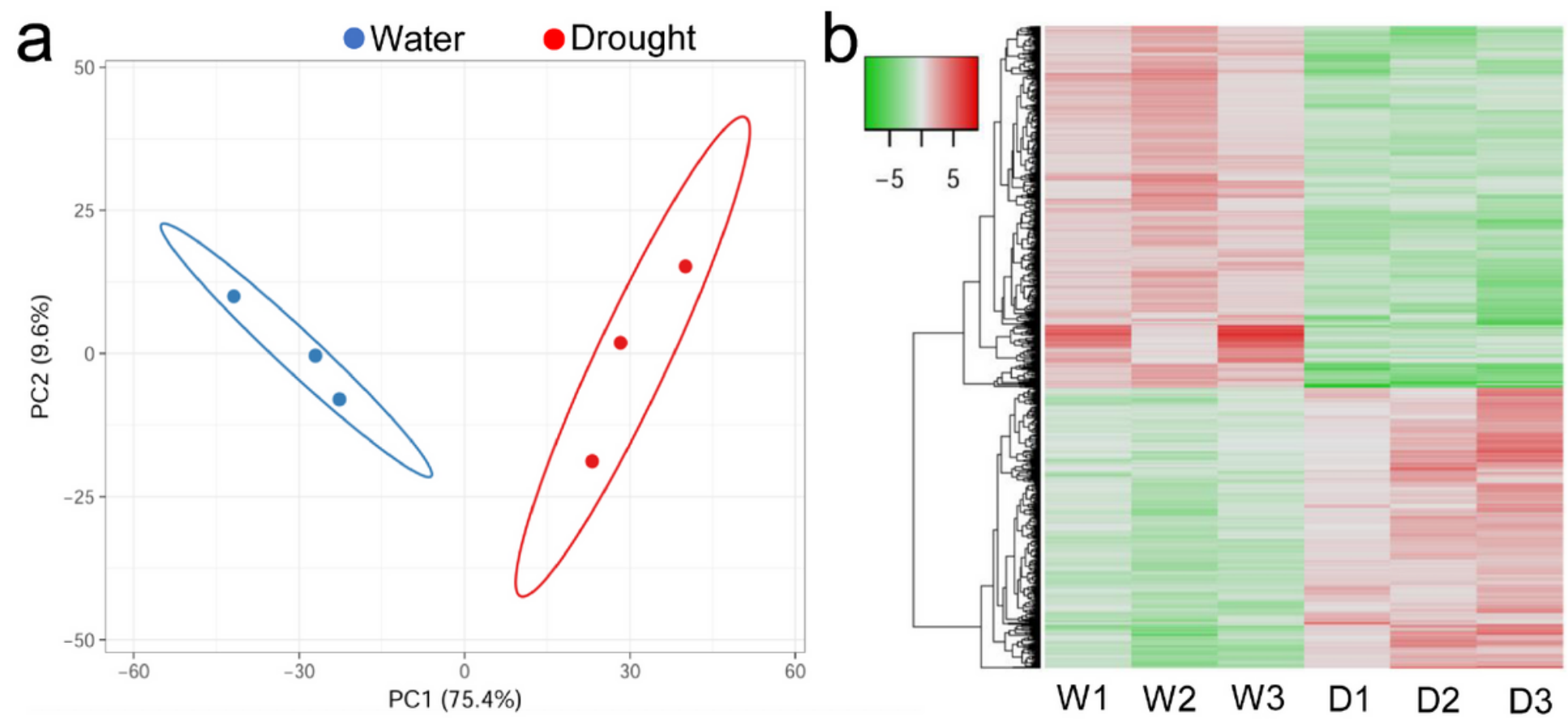

\section{Figure 3}

Principal component analysis (PCA) among samples of the experimental (Drought) and control (Water) groups in oil palm (a) and hierarchical clustering (b) of the relationships of samples between experimental (Drought) and control (Water) groups based on randomly selected DEGs. W1, W2 and W3 are controls, while D1, D2 and D3 are experimental samples. 


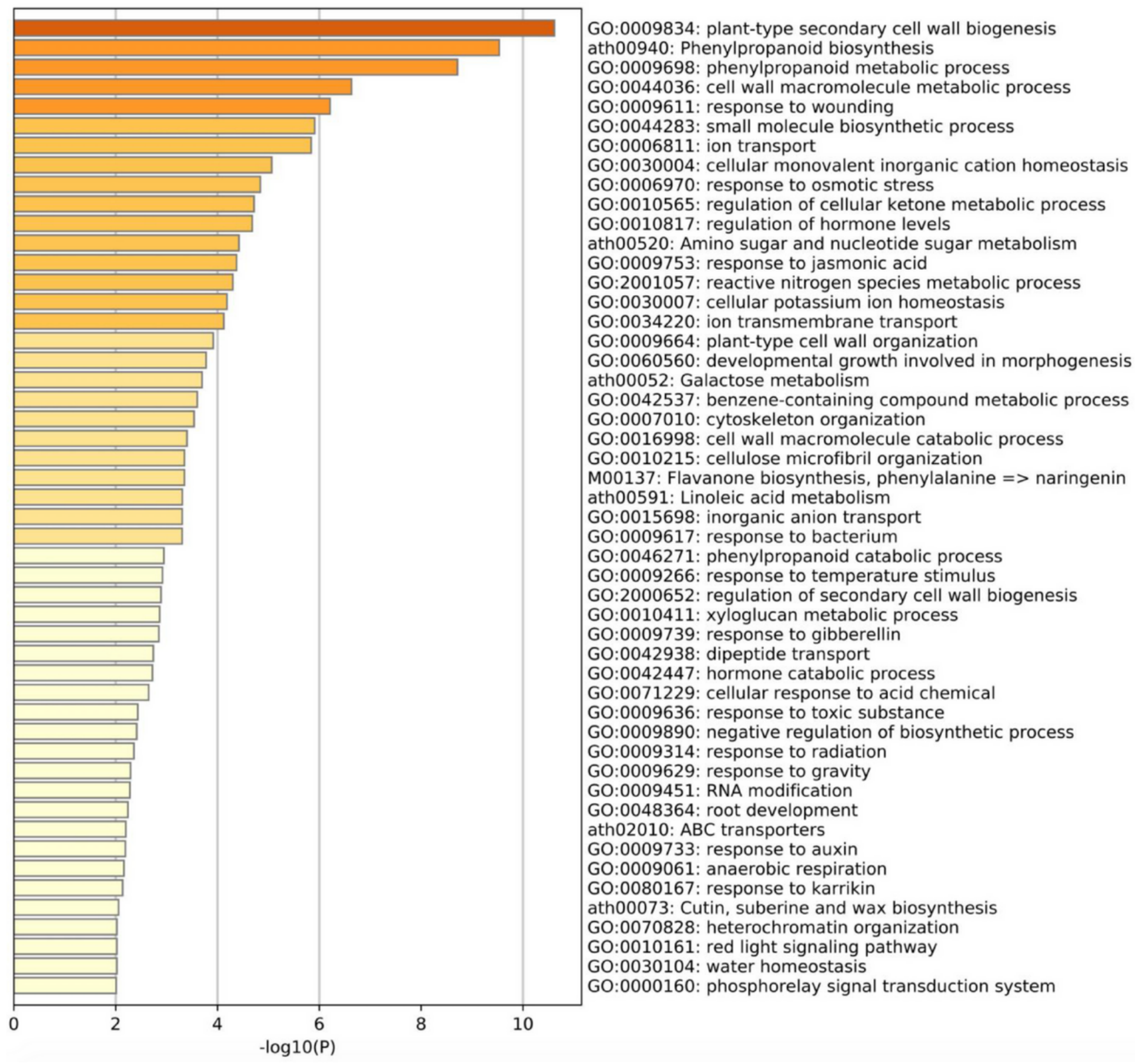

Figure 4

Enrichment of gene ontology (GO) of DEGs against drought challenge at the significance level of 0.01 in the roots of oil palm seedings under drought stress. 


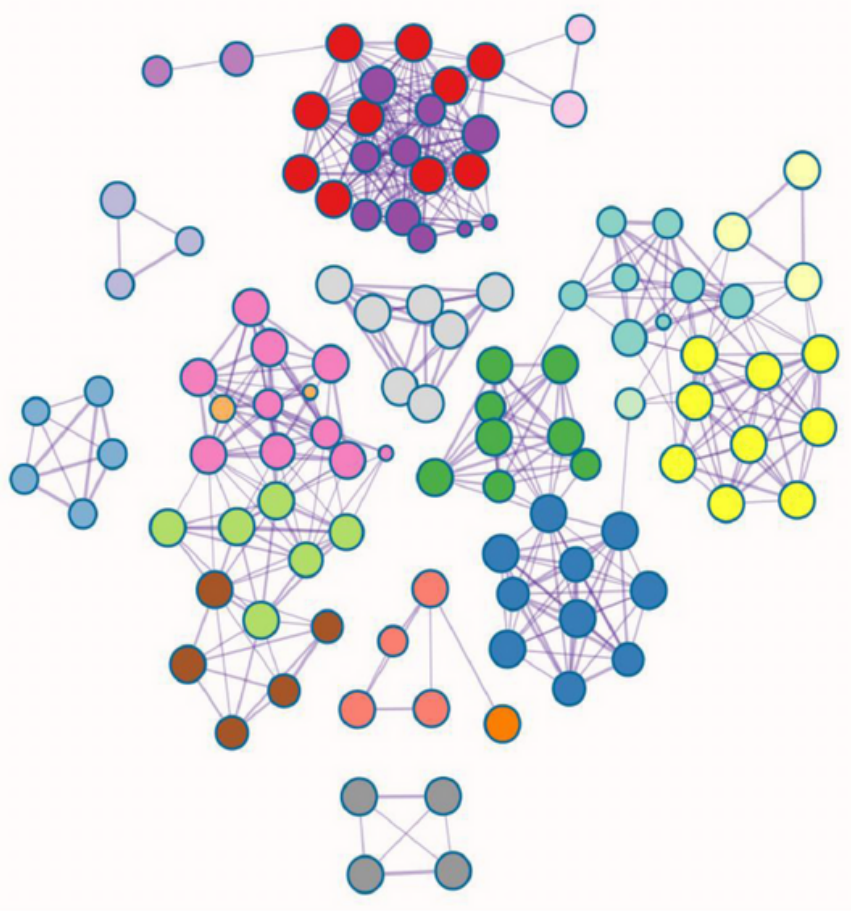

G0:0009834: plant-type secondary cell wall biogenesis ath00940: Phenylpropanoid biosynthesis G0:0009698: phenylpropanoid metabolic process G0:0044036: cell wall macromolecule metabolic process G0:0009611: response to wounding G0:0044283: small molecule biosynthetic process G0:0006811: ion transport G0:0030004: cellular monovalent inorganic cation homeostasis G0:0006970: response to osmotic stress G0:0010565: regulation of cellular ketone metabolic process G0:0010817: regulation of hormone levels ath00520: Amino sugar and nucleotide sugar metabolism G0:0009753: response to jasmonic acid GO:2001057: reactive nitrogen species metabolic process G0:0030007: cellular potassium ion homeostasis G0:0034220: ion transmembrane transport G0:0009664: plant-type cell wall organization G0:0060560: developmental growth involved in morphogenesis ath00052: Galactose metabolism G0:0042537: benzene-containing compound metabolic process

\section{Figure 5}

Major gene networks among the top 20 enriched GO terms as shown in Figure 5, based on DEGs against drought challenge in the roots of oil palm seedlings. Each network and the corresponding GO term are indicated with the same color. 


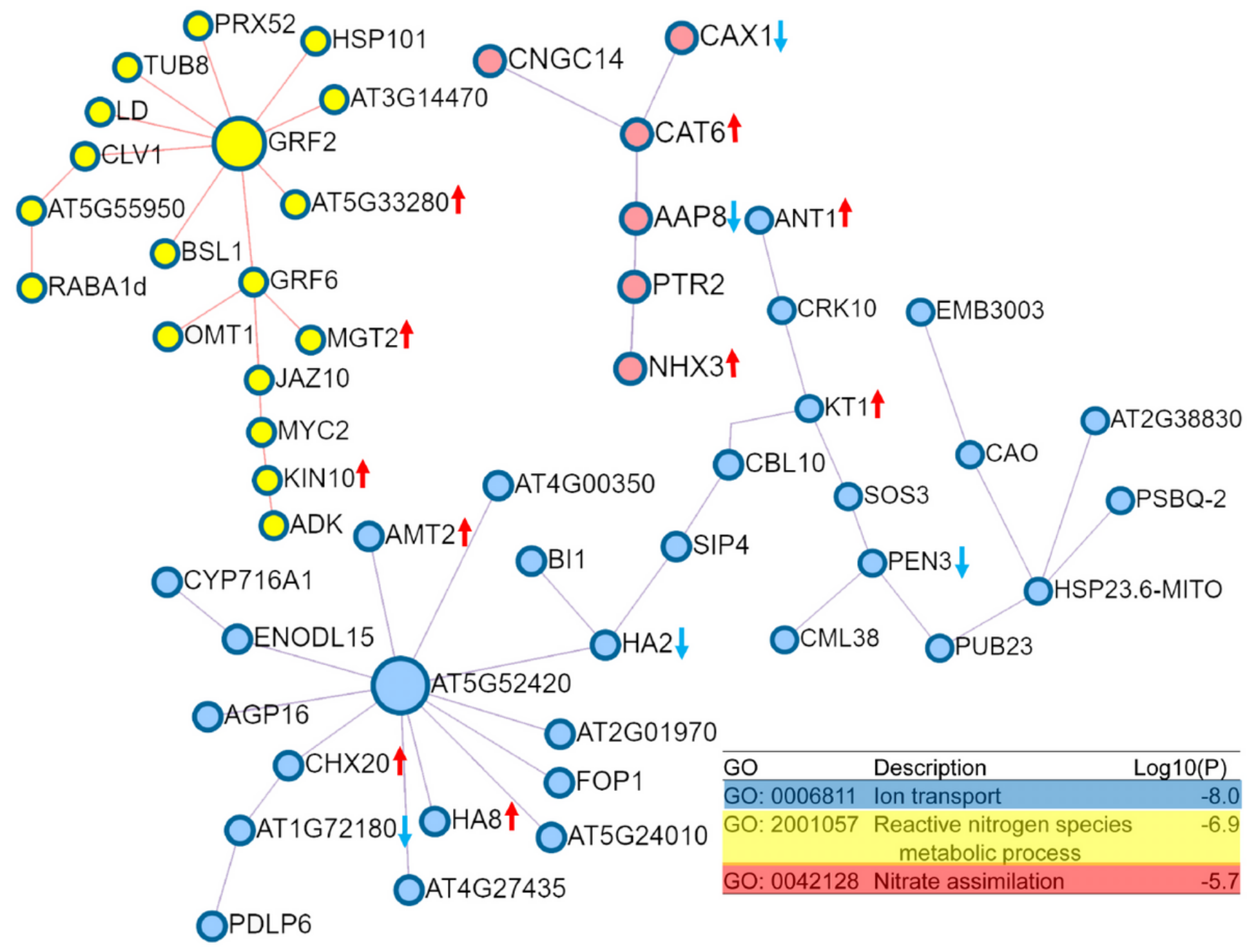

\section{Figure 6}

Three significant enrichments of protein-protein interaction networks identified based on DEGs against drought challenge in the roots of oil palm seedlings. DEGs in the three networks are further enriched with GO terms, with each showing corresponding significance. The networks are shown in different colors. The DEGs within each network are indicated and the expression patterns are shown with colored up- and down-arrows.

\section{Supplementary Files}

This is a list of supplementary files associated with this preprint. Click to download.

- SupplementaryTables123456.xlsx

- SupplementaryFigure1.pdf 